\title{
Characterization of the Linear Failure Rate Distribution by General Progressively Type-II Right Censored Order Statistics
}

\author{
M. M. Mohie El-Din ${ }^{1}$, A. Sadek ${ }^{1}$, Marwa M. Mohie El-Din ${ }^{2}$, A. M. Sharawy ${ }^{2, ~ * ~}$ \\ ${ }^{1}$ Department of Mathematics, Faculty of Science (Men), Al-Azhar University, Cairo, Egypt \\ ${ }^{2}$ Department of Mathematics, Faculty of Engineering, Egyptian Russian University, Cairo, Egypt
}

Email address:

ali.elsharawy82@yahoo.com (A. M. Sharawy)

${ }^{*}$ Corresponding author

\section{To cite this article:}

M. M. Mohie El-Din, A. Sadek, Marwa M. Mohie El-Din, A. M. Sharawy. Characterization of the Linear Failure Rate Distribution by General Progressively Type-II Right Censored Order Statistics. American Journal of Theoretical and Applied Statistics. Vol. 6, No. 3, 2017, pp. 129-140. doi: 10.11648/j.ajtas.20170603.11

Received: March 4, 2017; Accepted: March 20, 2017; Published: April 11, 2017

\begin{abstract}
In this article, we establish recurrence relations among single moments and among product moments of general progressively Type-II right censored order statistics and characterization for linear failure rate distribution using recurrence relations of single moments and product moments of general progressively Type-II right censored order statistics. Further, the results are specialized to the progressively Type-II right censored order statistics.
\end{abstract}

Keywords: Characterization, General Progressively Type-II Right Censored, Linear Failure Rate, Order Statistics, Recurrence Relations, Single and Product Moments

\section{Introduction}

In failure data analysis, it is common that some individuals cannot be observed for the full time to failure. the progressive Type-II right censored is a useful and more general scheme in which a specific fraction of individuals at risk may be removed from the study at each of several ordered failure times. Progressively censored samples have been considered, among others, by Balakrishnan et al. [6] and Davis and Feldstein [8]. Bain [5] derived analysis for the Linear Failure-Rate life-testing distribution. Aggarwala and Balakrishnan [3] derived recurrence relations for single and product moments of progressive Type-II right censored order statistics from exponential, Pareto and power function distributions and their truncated forms. Abd El-Aty and Marwa Mohie El-Din [1] derived recurrence relations for single and double moments of GOS from the inverted linear exponential distribution and any continuous function. Mokhlis et al. [13] derived recurrence relations for moments of GOS from Marshall-Olkin-Extended burr XII distribution. Mohie El-Din, and Kotb [12] derived recurrence relations for single and product moments of generalized order statistics for modified burr XII-Geometric distribution and characterization. Mohie El-Din et al. [11] derived Statistical Inference and Characterizations from Independent and Identical Exponential-Bernoulli Mixture Distribution. Athar et al. [4] discussed some new moments of progressively Type-II right censored order statistics from Lindley distribution. Saran and Pushkarna [9] derived moments of progressive Type-II right censored order statistics from a general class of doubly truncated continuous distributions. Abd El-Hamid et al. [2] derived inference and optimal design based on step-partially accelerated life tests for the generalized Pareto distribution under progressive Type-I censoring.

This scheme of censoring was generalized by Balakrishnan and Sandhu [7] as follows: at time $X_{0} \equiv 0, n$ units are placed on test; the first $r$ failure times, $X_{1}, \ldots, X_{r}$, are not observed; at time $X_{i}+0$, where $X_{i}$ is the $i^{t h}$ ordered failure time $(i=r+1, \ldots, m-1), R_{i}$ units are removed from the test randomly, so prior to the $(i+1)^{t h}$ failure there are $n_{i}=$ $n-i-\sum_{j=r+1}^{i} R_{j}$ units on test; finally, at the time of the $m^{\text {th }}$ failure, $X_{m}$, the experiment is terminated, i.e., the remaining $R_{m}$ units are removed from the test. The $R_{i}$ 's, $m$ 
and $r$ are prespecified integers which must satisfy the conditions: $\quad 0 \leq r<m \leq n, 0 \leq R_{i} \leq n_{i-1}$ for $i=r+$ $1, \ldots, m-1$ with $n_{r}=n-r$ and $R_{m}=n_{m-1}-1$.

The joint probability density function of the general progressively Type-II right censored order statistics failure times $X_{r+1: m:, n}, X_{r+2: m: n}, \ldots, X_{m: m: n}$, is given by

$$
\begin{gathered}
f_{X_{r+1: m: n}, \ldots, X_{m: m: n}}\left(x_{r+1}, \ldots, x_{m}\right)= \\
A_{(n, m-1)}\left[F\left(x_{r+1}, \theta\right)\right]^{r} \prod_{i=r+1}^{m} f\left(x_{i}, \theta\right)\left[1-F\left(x_{i}, \theta\right)\right]^{R_{i}} \\
x_{r+1}<x_{r+2}<\cdots<x_{m}
\end{gathered}
$$

where,

$$
\begin{gathered}
A_{(n, m-1)}=\frac{n !}{r !(n-r) !}\left(\prod_{j=r}^{m-1} n_{j}\right), n_{i}=n-i-\sum_{j=r+1}^{i} R_{j}, \\
i=r+1, \ldots, m-1 .
\end{gathered}
$$

In this paper, we shall introduce recurrence relations among single and product moments of general progressively Type-II right censored order statistics. Characterization for linear failure rate distribution using recurrence relations of

$$
\begin{gathered}
\left.\mu_{q: m: n}^{\left(R_{r+1}, R_{r+2}, \ldots, R_{m}\right)}\right)^{(i)}=E\left[X_{q: m: n}^{\left(R_{r+1}, R_{r+2}, \ldots, R_{m}\right)}\right]^{i} \\
=A_{(n, m-1)} \iint \cdots \int_{0<x_{r+1}<\cdots<x_{m}<\infty} x_{q}^{i}\left[F\left(x_{r+1}\right)\right]^{r} f\left(x_{r+1}\right)\left[1-F\left(x_{r+1}\right)\right]^{R_{r+1}} \times \\
f\left(x_{r+2}\right)\left[1-F\left(x_{r+2}\right)\right]^{R_{r+2}} \ldots f\left(x_{m}\right)\left[1-F\left(x_{m}\right)\right]^{R_{m}} d x_{r+1} \ldots d x_{m}
\end{gathered}
$$

and the $i^{\text {th }}$ and $j^{\text {th }}$ product moments as

$$
\begin{gathered}
\mu_{q, s: m: n}^{\left(R_{r+1}, R_{r+2}, \ldots, R_{m}\right)^{(i, j)}}=E\left[X_{q: m: n}^{\left(R_{r+1}, R_{r+2}, \ldots, R_{m}\right)^{i}} X_{s: m: n}^{\left(R_{r+1}, R_{r+2}, \ldots, R_{m}\right)^{j}}\right]= \\
A_{(n, m-1)} \iint \ldots \int_{0<x_{r+1}<\cdots<x_{m}<\infty} x_{q}^{i} x_{s}^{j}\left[F\left(x_{r+1}\right)\right]^{r} f\left(x_{r+1}\right)\left[1-F\left(x_{r+1}\right)\right]^{R_{r+1}} \times \\
f\left(x_{r+2}\right)\left[1-F\left(x_{r+2}\right)\right]^{R_{r+2}} \ldots f\left(x_{m}\right)\left[1-F\left(x_{m}\right)\right]^{R_{m}} d x_{r+1} \ldots d x_{m}
\end{gathered}
$$

\section{Recurrence Relations for Single and Product Moments}

In this section we introduce the recurrence relation for single and product moments of general progressively Type-II right censored order statistics from linear failure rate

$$
\begin{gathered}
\mu_{q: m: n}^{\left(R_{r+1}, \ldots, R_{m}\right)^{(i+2)}}=\frac{(i+2)}{\theta\left(R_{q}+1\right)} \mu_{q: m: n}^{\left(R_{r+1}, \ldots, R_{m}\right)^{(i)}}-\frac{\alpha(i+2)}{\theta(i+1)} \mu_{q: m: n}^{\left(R_{r+1}, \ldots, R_{m}\right)^{(i+1)}} \\
+\frac{\left(n-R_{r+1}-\cdots-R_{q-1}-q+1\right)}{\left(R_{q}+1\right)}\left[\mu_{q-1: m-1: n}^{\left(R_{r+1}, \ldots, R_{q-2},\left(R_{q-1}+R_{q}+1\right), R_{q+1}, \ldots, R_{m}\right)^{(i+2)}}+\frac{\alpha(i+2)}{\theta(i+1)} \mu_{q-1: m-1: n}^{\left(R_{r+1}, \ldots, R_{q-2},\left(R_{q-1}+R_{q}+1\right), R_{q+1}, \ldots, R_{m}\right)^{(i+1)}}\right] \\
\left.-\frac{\left(n-R_{r+1}-\cdots-R_{q}-q\right)}{\left(R_{q}+1\right)}\left[\mu_{q: m-1: n}^{\left(R_{r+1}, \ldots, R_{q-1},\left(R_{q}+R_{q+1}+1\right), R_{q+2}, \ldots, R_{m}\right)^{(i+2)}}+\frac{\alpha(i+2)}{\theta(i+1)} \mu_{q: m-1: n}^{\left(R_{r+1}, \ldots, R_{q-1},\left(R_{q}+R_{q+1}+1\right), R_{q+2}, \ldots, R_{m}\right)}\right)^{(i+1)}\right]
\end{gathered}
$$


Proof

From Eq. (4) and Eq. (5), we get

$$
\begin{gathered}
\mu_{q: m: n}^{\left(R_{r+1}, \ldots, R_{m}\right)^{(i)}}=A_{(n, m-1)} \iint \ldots \int_{0<x_{r+1}<\cdots<x_{q-1}<x_{q+1}<\cdots<x_{m}<\infty} I_{1}\left(x_{q-1}, x_{q+1}\right)\left[F\left(x_{r+1}\right)\right]^{r} \times \\
f\left(x_{r+1}\right)\left[1-F\left(x_{r+1}\right)\right]^{R_{r+1}} \ldots f\left(x_{q-1}\right)\left[1-F\left(x_{q-1}\right)\right]^{R_{q-1}} f\left(x_{q+1}\right)\left[1-F\left(x_{q+1}\right)\right]^{R_{q+1}} \ldots \times \\
f\left(x_{m}\right)\left[1-F\left(x_{m}\right)\right]^{R_{m}} d x_{r+1} d x_{r+2} \ldots d x_{q-1} d x_{q+1} \ldots d x_{m}
\end{gathered}
$$

where

$$
I_{1}\left(x_{q-1}, x_{q+1}\right)=\int_{x_{q-1}}^{x_{q+1}} x_{q}^{i}\left(\alpha+\theta x_{q}\right)\left[1-F\left(x_{q}\right)\right]^{R_{q}+1} d x_{q}
$$

Now, integrating by parts gives

$$
\begin{gathered}
I_{1}\left(x_{q-1}, x_{q+1}\right)= \\
F\left(x_{q+1}^{i+1}\left[1-F\left(x_{q+1}\right)\right]^{R q} d x_{q}+\frac{\theta\left(R_{q}+1\right)}{i+2} \int_{x_{q-1}}^{x_{q+1}} x_{q}^{i+2} f\left(x_{q}\right)\left[1-F\left(x_{q-1}\right)\right]^{R q} d x_{q}\right. \\
i+1
\end{gathered}
$$

Substituting Eq. (10) in Eq. (8) and simplifying, yields Eq. (7).

This completes the proof.

Special case

$$
\begin{gathered}
\mu_{q: m: n}^{\left(R_{1}, \ldots, R_{m}\right)^{(i+2)}}=\frac{(i+2)}{\theta\left(R_{q}+1\right)} \mu_{q: m: n}^{\left(R_{1}, \ldots, R_{m}\right)^{(i)}}-\frac{\alpha(i+2)}{\theta(i+1)} \mu_{q: m: n}^{\left(R_{1}, \ldots, R_{m}\right)^{(i+1)}} \\
+\frac{\left(n-R_{1}-\cdots-R_{q-1}-q+1\right)}{\left(R_{q}+1\right)}\left[\mu_{q-1: m-1: n}^{\left(R_{1}, \ldots, R_{q-2},\left(R_{q-1}+R_{q}+1\right), R_{q+1}, \ldots, R_{m}\right)^{(i+2)}}+\frac{\alpha(i+2)}{\theta(i+1)} \mu_{q-1: m-1: n}^{\left(R_{1}, \ldots, R_{q-2},\left(R_{q-1}+R_{q}+1\right), R_{q+1}, \ldots, R_{m}\right)^{(i+1)}}\right] \\
\left.-\frac{\left(n-R_{1}-\cdots-R_{q}-q\right)}{\left(R_{q}+1\right)}\left[\mu_{q: m-1: n}^{\left(R_{1}, \ldots, R_{q-1},\left(R_{q}+R_{q+1}+1\right), R_{q+2}, \ldots, R_{m}\right)}\right)^{(i+2)}+\frac{\alpha(i+2)}{\theta(i+1)} \mu_{q: m-1: n}^{\left(R_{1}, \ldots, R_{q-1},\left(R_{q}+R_{q+1}+1\right), R_{q+2}, \ldots, R_{m}\right)^{(i+1)}}\right]
\end{gathered}
$$

In the next two theorems, we introduce recurrence relations for product moments of general progressively TypeII right censored order statistics from linear failure rate
Theorem (2.1) will be valid for the progressively Type-II right censored order statistics as a special case from the general progressively Type-II right censored order statistics when $r=0$,

$$
\begin{gathered}
\mu_{q, s: m: n}^{\left(R_{r+1}, \ldots, R_{m}\right)^{(i+2, j)}}=\frac{(i+2)}{\theta\left(R_{q}+1\right)} \mu_{q, s: m: n}^{\left(R_{r+1}, \ldots, R_{m}\right)^{(i, j)}}-\frac{\alpha(i+2)}{\theta(i+1)} \mu_{q, s: m: n}^{\left(R_{r+1}, \ldots, R_{m}\right)^{(i+1, j)}} \\
+\frac{\left(n-R_{r+1}-\cdots-R_{q-1}-q+1\right)}{\left(R_{q}+1\right)}\left[\mu_{q-1, s-1: m-1: n}^{\left(R_{r+1}, \ldots, R_{q-2},\left(R_{q-1}+R_{q}+1\right), R_{q+1}, \ldots, R_{m}\right)^{(i+2, j)}}\right. \\
\left.+\frac{\alpha(i+2)}{\theta(i+1)} \mu_{q-1, s-1: m-1: n}^{\left(R_{r+1}, \ldots, R_{q-2},\left(R_{q-1}+R_{q}+1\right), R_{q+1}, \ldots, R_{m}\right)^{(i+1, j)}}\right] \\
-\frac{\left(n-R_{r+1}-\cdots-R_{q}-q\right)}{\left(R_{q}+1\right)}\left[\mu_{q, s-1: m-1: n}^{\left(R_{r+1}, \ldots, R_{q-1},\left(R_{q}+R_{q+1}+1\right), R_{q+2}, \ldots, R_{m}\right)^{(i+2, j)}}+\frac{\alpha(i+2)}{\theta(i+1)} \mu_{q, s-1: m-1: n}^{\left(R_{r+1}, \ldots, R_{q-1},\left(R_{q}+R_{q+1}+1\right), R_{q+2}, \ldots, R_{m}\right)^{(i+1, j)}}\right]
\end{gathered}
$$

Proof

From Eq. (6), we get

$$
\begin{aligned}
& \left.\mu_{q, s: m: n}^{\left(R_{r+1}, \ldots, R_{m}\right.}\right)^{(i, j)}=A_{(n, m-1)} \iint \ldots \int_{0<x_{r+1}<\cdots<x_{q-1}<x_{q+1}<\cdots<x_{m}<\infty} x_{s}^{j} I_{1}\left(x_{q-1}, x_{q+1}\right)\left[F\left(x_{r+1}\right)\right]^{r} \\
& \times f\left(x_{r+1}\right)\left[1-F\left(x_{r+1}\right)\right]^{R_{r+1}} \ldots f\left(x_{q-1}\right)\left[1-F\left(x_{q-1}\right)\right]^{R_{q-1}} f\left(x_{q+1}\right)\left[1-F\left(x_{q+1}\right)\right]^{R_{q+1}} \ldots \times
\end{aligned}
$$




$$
f\left(x_{m}\right)\left[1-F\left(x_{m}\right)\right]^{R_{m}} d x_{r+1} d x_{r+2} \ldots d x_{q-1} d x_{q+1} \ldots d x_{m}
$$

Substituting by Eq. (10) in Eq. (12) and simplifying, yields Eq. (11).

This completes the proof.

Special case
Theorem (2.2) will be valid for the progressively Type-II right censored order statistics as a special case from the general progressively Type-II right censored order statistics when $r=0$,

$$
\begin{gathered}
\mu_{q, s: m: n}^{\left(R_{1}, \ldots, R_{m}\right)^{(i+2, j)}}=\frac{(i+2)}{\theta\left(R_{q}+1\right)} \mu_{q, s: m: n}^{\left(R_{1}, \ldots, R_{m}\right)^{(i, j)}}-\frac{\alpha(i+2)}{\theta(i+1)} \mu_{q, s: m: n}^{\left(R_{1}, \ldots, R_{m}\right)^{(i+1, j)}} \\
+\frac{\left(n-R_{1}-\cdots-R_{q-1}-q+1\right)}{\left(R_{q}+1\right)}\left[\mu_{q-1, s-1: m-1: n}^{\left(R_{1}, \ldots, R_{q-2},\left(R_{q-1}+R_{q}+1\right), R_{q+1}, \ldots, R_{m}\right)^{(i+2, j)}}+\frac{\alpha(i+2)}{\theta(i+1)} \mu_{q-1, s-1: m-1: n}^{\left(R_{1}, \ldots, R_{q-2},\left(R_{q-1}+R_{q}+1\right), R_{q+1}, \ldots, R_{m}\right)^{(i+1, j)}}\right] \\
-\frac{\left(n-R_{1}-\cdots-R_{q}-q\right)}{\left(R_{q}+1\right)}\left[\mu_{q, s-1: m-1: n}^{\left(R_{1}, \ldots, R_{q-1},\left(R_{q}+R_{q+1}+1\right), R_{\left.q+2, \ldots, R_{m}\right)}\right)^{(i+2, j)}}+\frac{\alpha(i+2)}{\theta(i+1)} \mu_{q, s-1: m-1: n}^{\left(R_{1}, \ldots, R_{q-1},\left(R_{q}+R_{q+1}+1\right), R_{q+2}, \ldots, R_{m}\right)^{(i+1, j)}}\right]
\end{gathered}
$$

Theorem 2.3

For $r+1 \leq q<s \leq m-1, m \leq n$ and $i, j \geq 0$, then

$$
\begin{aligned}
& \mu_{q, s: m: n}^{\left(R_{r+1}, \ldots, R_{m}\right)^{(i, j+2)}}=\frac{(j+2)}{\theta\left(R_{s}+1\right)} \mu_{q, s: m: n}^{\left(R_{r+1}, \ldots, R_{m}\right)^{(i, j)}}-\frac{\alpha(j+2)}{\theta(j+1)} \mu_{q, s: m: n}^{\left(R_{r+1}, \ldots, R_{m}\right)^{(i, j+1)}} \\
& +\frac{\left(n-R_{r+1}-\cdots-R_{s-1}-s+1\right)}{\left(R_{S}+1\right)}\left[\mu_{q, S-1: m-1: n}^{\left(R_{r+1}, \ldots, R_{s-2},\left(R_{S-1}+R_{S}+1\right), R_{S+1}, \ldots, R_{m}\right)^{(i, j+2)}}+\frac{\alpha(j+2)}{\theta(j+1)} \mu_{q, S-1: m-1: n}^{\left(R_{r+1}, \ldots, R_{S-2},\left(R_{S-1}+R_{S}+1\right), R_{S+1}, \ldots, R_{m}\right)^{(i, j+1)}}\right] \\
& -\frac{\left(n-R_{r+1}-\cdots-R_{S}-s\right)}{\left(R_{S}+1\right)}\left[\mu_{q, s: m-1: n}^{\left(R_{r+1}, \ldots, R_{S-1},\left(R_{S}+R_{S+1}+1\right), R_{S+2}, \ldots, R_{m}\right)^{(i, j+2)}}+\frac{\alpha(j+2)}{\theta(j+1)} \mu_{q, s: m-1: n}^{\left(R_{r+1}, \ldots, R_{S-1},\left(R_{S}+R_{S+1}+1\right), R_{S+2}, \ldots, R_{m}\right)^{(i, j+1)}}\right]
\end{aligned}
$$

Proof

From Eq. (4) and Eq. (6), we get

$$
\begin{gathered}
\left.\mu_{q, s: m: n}^{\left(R_{r+1}, \ldots, R_{m}\right)}\right)^{(i, j)}=A_{(n, m-1)} \iint \ldots \int_{0<x_{r+1}<\cdots<x_{s-1}<x_{s+1}<\cdots<x_{m}<\infty} x_{q}^{i} I_{2}\left(x_{s-1}, x_{s+1}\right)\left[F\left(x_{r+1}\right)\right]^{r} \\
\times f\left(x_{r+1}\right)\left[1-F\left(x_{r+1}\right)\right]^{R_{r+1}} \ldots f\left(x_{s-1}\right)\left[1-F\left(x_{s-1}\right)\right]^{R_{s-1}} f\left(x_{s+1}\right)\left[1-F\left(x_{s+1}\right)\right]^{R_{s+1}} \ldots \times \\
f\left(x_{m}\right)\left[1-F\left(x_{m}\right)\right]^{R_{m}} d x_{r+1} d x_{r+2} \ldots d x_{s-1} d x_{s+1} \ldots d_{m}
\end{gathered}
$$

where

$$
I_{2}\left(x_{s-1}, x_{s+1}\right)=\int_{x_{s-1}}^{x_{s+1}} x_{s}^{j}\left(\alpha+\theta x_{s}\right)\left[1-F\left(x_{s}\right)\right]^{R_{s}+1} d x_{s}
$$

Now, integrating by parts we obtain

$$
\begin{gathered}
\left\{\frac{\alpha x_{S+1}^{j+1}\left[1-F\left(x_{S+1}\right)\right]^{R_{S}+1}-\alpha x_{s-1}^{j+1}\left[1-F\left(x_{S-1}\right)\right]^{R_{S}+1}}{j+1}+\frac{\theta x_{s+1}^{j+2}\left[1-F\left(x_{S+1}\right)\right]^{R_{S}+1}-\theta x_{s-1}^{j+2}\left[1-F\left(x_{S-1}\right)\right]^{R_{S}+1}}{j+2}+\frac{\alpha\left(R_{S}+1\right)}{j+1} \int_{x_{S-1}}^{x_{S+1}} x_{s}^{j+1} f\left(x_{s}\right)[1-\right. \\
\left.\left.F\left(x_{S}\right)\right]^{R_{S}} d x_{S}+\frac{\theta\left(R_{S}+1\right)}{j+2} \int_{x_{S-1}}^{x_{S+1}} x_{S}^{j+2} f\left(x_{S}\right)\left[1-F\left(x_{S}\right)\right]^{R_{S}} d x_{s}\right\}
\end{gathered}
$$

Substituting by Eq. (16) in Eq. (14) and simplifying, yields Eq. (13).

This completes the proof.

$$
\begin{aligned}
& \mu_{q, s: m: n}^{\left(R_{1}, \ldots, R_{m}\right)^{(i, j+2)}}=\frac{(j+2)}{\theta\left(R_{S}+1\right)} \mu_{q, s: m: n}^{\left(R_{1}, \ldots, R_{m}\right)^{(i, j)}}-\frac{\alpha(j+2)}{\theta(j+1)} \mu_{q, s: m: n}^{\left(R_{1}, \ldots, R_{m}\right)^{(i, j+1)}} \\
& +\frac{\left(n-R_{1}-\cdots-R_{S-1}-s+1\right)}{\left(R_{S}+1\right)}\left[\mu_{q, s-1: m-1: n}^{\left(R_{1}, \ldots, R_{s-2,}\left(R_{S-1}+R_{S}+1\right), R_{S+1}, \ldots, R_{m}\right)^{(i, j+2)}}+\frac{\alpha(j+2)}{\theta(j+1)} \mu_{q, s-1: m-1: n}^{\left(R_{1}, \ldots, R_{S-2},\left(R_{S-1}+R_{S}+1\right), R_{S+1}, \ldots, R_{m}\right)^{(i, j+1)}}\right]
\end{aligned}
$$

Theorem (2.2) will be valid for the progressively Type-II right censored order statistics as a special case from the general progressively Type-II right censored order statistics when $r=0$, 


$$
-\frac{\left(n-R_{1}-\cdots-R_{s}-s\right)}{\left(R_{S}+1\right)}\left[\mu_{q, s: m-1: n}^{\left(R_{1}, \ldots, R_{S-1},\left(R_{S}+R_{S+1}+1\right), R_{S+2}, \ldots, R_{m}\right)^{(i, j+2)}}+\frac{\alpha(j+2)}{\theta(j+1)} \mu_{q, s: m-1: n}^{\left(R_{1}, \ldots, R_{S-1},\left(R_{S}+R_{S+1}+1\right), R_{S+2}, \cdots, R_{m}\right)^{(i, j+1)}}\right]
$$

\section{Characterization for Single and Product Moments}

In this section, we introduce the characterization of the linear failure rate distribution using the relation between pdf and cdf and using recurrence relation for single and product moments of general progressively Type-II right censored order statistics from linear failure rate distribution.

In the next theorem, we introduce the characterization of the linear failure rate distribution using relation between pdf and cdf.

Theorem 3.1

Let $X$ be a continuous random variable with $\operatorname{pdf} f(\cdot)$, cdf $F(\cdot)$ and survival function $[1-F(\cdot)]$. Then $X$ has linear failure rate distribution iff

$$
f(x)=[\alpha+\theta x][1-F(x)] \cdot x \geq 0
$$

Proof

Necessity:

From Eq. (2) and Eq. (3) we can easily obtain Eq. (17).

Sufficiency:

Suppose that $X$ is a continuous random variable with pdf $f(\cdot)$ and $\operatorname{cdf} F(\cdot)$. Suppose, also, that equation Eq. (17) is true. Then we have:

$$
\begin{aligned}
& \frac{-d[1-F(x)]}{1-F(x)}=[\alpha+\theta x] d x \\
& \mu_{q: m: n}^{\left(R_{r+1}, \ldots, R_{m}\right)^{(i+2)}}=\frac{(i+2)}{\theta\left(R_{q}+1\right)} \mu_{q: m: n}^{\left(R_{r+1}, \ldots, R_{m}\right)^{(i)}}-\frac{\alpha(i+2)}{\theta(i+1)} \mu_{q: m: n}^{\left(R_{r+1}, \ldots, R_{m}\right)^{(i+1)}} \\
& +\frac{\left(n-R_{r+1}-\cdots-R_{q-1}-q+1\right)}{\left(R_{q}+1\right)}\left[\mu_{q-1: m-1: n}^{\left(R_{r+1}, \ldots, R_{q-2},\left(R_{q-1}+R_{q}+1\right), R_{q+1}, \ldots, R_{m}\right)^{(i+2)}}+\frac{\alpha(i+2)}{\theta(i+1)} \mu_{q-1: m-1: n}^{\left(R_{r+1}, \ldots, R_{q-2},\left(R_{q-1}+R_{q}+1\right), R_{q+1}, \ldots, R_{m}\right)^{(i+1)}}\right] \\
& -\frac{\left(n-R_{r+1}-\cdots-R_{q}-q\right)}{\left(R_{q}+1\right)}\left[\mu_{q: m-1: n}^{\left(R_{r+1}, \ldots, R_{q-1},\left(R_{q}+R_{q+1}+1\right), R_{q+2}, \ldots, R_{m}\right)^{(i+2)}}+\frac{\alpha(i+2)}{\theta(i+1)} \mu_{q: m-1: n}^{\left(R_{r+1}, \ldots, R_{q-1},\left(R_{q}+R_{q+1}+1\right), R_{q+2}, \ldots, R_{m}\right)^{(i+1)}}\right]
\end{aligned}
$$

Proof

Necessity:

Theorem 2.1 proved the necessary part of this theorem. where $\mathrm{C}$ is an arbitrary constant.

Now, since $[1-F(0)]=1$, then putting $x=0$ in this equation, we get $C=0$.

Therefore,

$$
-\ln |1-F(x)|=\alpha x+\frac{\theta}{2} x^{2}
$$

Hence,

$$
F(x)=1-e^{-\alpha x-\frac{\theta}{2} x^{2}} .
$$

That is the distribution function of linear failure rate distribution.

This completes the proof.

In the next theorem, we introduce the characterization of the linear failure rate distribution using recurrence relation for single moment of general progressively Type-II right censored order statistics has introduced in the following theorems.

Theorem 3.2

Let $X_{r+1: n} \leq X_{r+2: n} \leq \ldots \leq X_{m: n}$ be the order stasistics of a

$$
\begin{gathered}
\mu_{q: m: n}^{\left(R_{r+1}, \ldots, R_{m}\right)^{(i)}}=\frac{\theta\left(R_{q}+1\right)}{(i+2)} \mu_{q: m: n}^{\left(R_{r+1}, \ldots, R_{m}\right)^{(i+2)}}+\frac{\alpha\left(R_{q}+1\right)}{(i+1)} \mu_{q: m: n}^{\left(R_{r+1}, \ldots, R_{m}\right)^{(i+1)}} \\
\left.+\left(n-R_{r+1}-\cdots-R_{q}-q\right)\left[\frac{\theta}{(i+2)} \mu_{q: m-1: n}^{\left(R_{r+1}, \ldots, R_{q-1},\left(R_{q}+R_{q+1}+1\right), R_{q+2}, \ldots, R_{m}\right)^{(i+2)}}+\frac{\alpha}{(i+1)} \mu_{q: m-1: n}^{\left(R_{r+1}, \ldots, R_{q-1},\left(R_{q}+R_{q+1}+1\right), R_{q+2}, \ldots, R_{m}\right)}\right)^{(i+1)}\right] \\
-\left(n-R_{r+1}-\cdots-R_{q-1}-q+1\right)\left[\frac{\theta}{(i+2)} \mu_{q-1: m-1: n}^{\left(R_{r+1}, \ldots, R_{q-2},\left(R_{q-1}+R_{q}+1\right), R_{q+1}, \ldots, R_{m}\right)^{(i+2)}}+\frac{\alpha}{(i+1)} \mu_{q-1: m-1: n}^{\left(R_{r+1}, \ldots, R_{q-2},\left(R_{q-1}+R_{q}+1\right), \ldots, R_{m}\right)^{(i+1)}}\right](19)
\end{gathered}
$$

From Eq. (4) and Eq. (5), we get 


$$
\begin{gathered}
\mu_{q: m: n}^{\left(R_{r+1}, \ldots, R_{m}\right)^{(i+1)}}=A_{(n, m-1)} \iint \ldots \int_{0<x_{r+1}<\cdots<x_{q-1}<x_{q+1}<\cdots<x_{m}<\infty} I_{3}\left(x_{q-1}, x_{q+1}\right)\left[F\left(x_{r+1}\right)\right]^{r} \times \\
f\left(x_{r+1}\right)\left[1-F\left(x_{r+1}\right)\right]^{R_{r+1}} \ldots f\left(x_{q-1}\right)\left[1-F\left(x_{q-1}\right)\right]^{R_{q-1}} f\left(x_{q+1}\right)\left[1-F\left(x_{q+1}\right)\right]^{R_{q+1}} \ldots \times \\
f\left(x_{m}\right)\left[1-F\left(x_{m}\right)\right]^{R_{m}} d x_{r+1} \ldots d x_{q-1} d x_{q+1} \ldots d x_{m}
\end{gathered}
$$

where

$$
I_{3}\left(x_{q-1}, x_{q+1}\right)=\int_{x_{q-1}}^{x_{q+1}} x_{q}^{i+1} f\left(x_{q}\right)\left[1-F\left(x_{q}\right)\right]^{R q} d x_{q}
$$

Integrating by parts, we obtain

$$
I_{3}\left(x_{q-1}, x_{q+1}\right)=\frac{-1}{R_{q}+1} x_{q+1}^{i+1}\left[1-F\left(x_{q+1}\right)\right]^{R_{q}+1}+\frac{1}{R_{q}+1} x_{q-1}^{i+1}\left[1-F\left(x_{q-1}\right)\right]^{R_{q}+1}+\frac{i+1}{R_{q}+1} \int_{x_{q-1}}^{x_{q+1}} x_{q}^{i}\left[1-F\left(x_{q}\right)\right]^{R_{q}+1} d x_{q}
$$

Substituting in Eq. (20), we get

$$
\begin{aligned}
& \mu_{q: m: n}^{\left(R_{r+1}, \cdots, R_{m}\right)}{ }^{(i+1)}=\frac{i+1}{R_{q}+1} A_{(n, m-1)} \iint \ldots \int_{0<x_{r+1}<\cdots<x_{q-1}<x_{q+1}<\cdots<x_{m}<\infty}\left[F\left(x_{r+1}\right)\right]^{r} \times \\
& f\left(x_{r+1}\right)\left[1-F\left(x_{r+1}\right)\right]^{R_{r+1}} \ldots f\left(x_{q-1}\right)\left[1-F\left(x_{q-1}\right)\right]^{R_{q-1}} \int_{x_{q-1}}^{x_{q+1}} x_{q}^{i}\left[1-F\left(x_{q}\right)\right]^{R_{q}+1} d x_{q} \\
& f\left(x_{q+1}\right)\left[1-F\left(x_{q+1}\right)\right]^{R q+1} \ldots f\left(x_{m}\right)\left[1-F\left(x_{m}\right)\right]^{R_{m}} d x_{r+1} \ldots d x_{q-1} d x_{q+1} \ldots d x_{m} \\
& +\frac{A_{(n, m-1)}}{R_{q}+1} \iint \cdots \int_{0<x_{r+1}<\cdots<x_{q-1}<x_{q+1}<\cdots<x_{m}<\infty} x_{q-1}^{i+1}\left[F\left(x_{r+1}\right)\right]^{r} \times \\
& f\left(x_{r+1}\right)\left[1-F\left(x_{r+1}\right)\right]^{R_{r+1}} \ldots f\left(x_{q-1}\right)\left[1-F\left(x_{q-1}\right)\right]^{1+R_{q q}+R_{q-1}} f\left(x_{q+1}\right)\left[1-F\left(x_{q+1}\right)\right]^{R_{q+1}} \\
& \times \ldots f\left(x_{m}\right)\left[1-F\left(x_{m}\right)\right]^{R_{m}} d x_{r+1} \ldots d x_{q-1} d x_{q+1} \ldots d x_{m} \\
& -\frac{A_{(n, m-1)}}{R_{q}+1} \iint \cdots \int_{0<x_{r+1}<\cdots<x_{q-1}<x_{q+1}<\cdots<x_{m}<\infty} x_{q+1}^{i+1}\left[F\left(x_{r+1}\right)\right]^{r} \times \\
& f\left(x_{r+1}\right)\left[1-F\left(x_{r+1}\right)\right]^{R_{r+1}} \ldots f\left(x_{q-1}\right)\left[1-F\left(x_{q-1}\right)\right]^{R_{q-1}} f\left(x_{q+1}\right)\left[1-F\left(x_{q+1}\right)\right]^{1+R_{q}+R_{q+1}} \\
& \times \ldots f\left(x_{m}\right)\left[1-F\left(x_{m}\right)\right]^{R_{m}} d x_{r+1} \ldots d x_{q-1} d x_{q+1} \ldots d x_{m} \\
& =A_{(n, m-1)} \frac{i+1}{R_{q}+1} \iint \cdots \int_{0<x_{r+1}<\cdots<x_{q-1}<x_{q+1}<\cdots<x_{m}<\infty}\left[F\left(x_{r+1}\right)\right]^{r} \times \\
& f\left(x_{r+1}\right)\left[1-F\left(x_{r+1}\right)\right]^{R r+1} \ldots f\left(x_{q-1}\right)\left[1-F\left(x_{q-1}\right)\right]^{R q-1} \int_{x_{q-1}}^{x_{q+1}} x_{q}^{i}\left[1-F\left(x_{q}\right)\right]^{R_{q}+1} d x_{q} \times \\
& f\left(x_{q+1}\right)\left[1-F\left(x_{q+1}\right)\right]^{R q+1} \ldots f\left(x_{m}\right)\left[1-F\left(x_{m}\right)\right]^{R_{m}} d x_{r+1} \ldots d x_{q-1} d x_{q+1} \ldots d x_{m}+ \\
& \frac{\left(n-R_{r+1}-\cdots-R_{q}-q\right)}{R_{q}+1} \mu_{q: m-1: n}^{\left(R_{r+1}, \ldots, R_{q-1},\left(R_{q}+R_{q+1}+1\right), R_{q+2}, \cdots, R_{m}\right)^{(i+1)}} \\
& \left.-\frac{\left(n-R_{r+1}-\cdots-R_{q-1}-q+1\right)}{R_{q}+1} \mu_{q-1: m-1: n}^{\left(R_{r+1}, \ldots R_{q-2},\left(R_{q-1}+R_{q}+1\right), R_{q+1}, \ldots, R_{m}\right)}\right)^{(i+1)}
\end{aligned}
$$

and 


$$
\begin{gathered}
\mu_{q: m: n}^{\left(R_{r+1, \ldots, R_{m}}\right)^{(i+2)}}=A_{(n, m-1)} \frac{i+2}{R_{q}+1} \iint \ldots \int_{0<x_{r+1}<\cdots<x_{q-1}<x_{q+1}<\cdots<x_{m}<\infty}\left[F\left(x_{r+1}\right)\right]^{r} \times \\
f\left(x_{r+1}\right)\left[1-F\left(x_{r+1}\right)\right]^{R_{r+1}} \ldots f\left(x_{q-1}\right)\left[1-F\left(x_{q-1}\right)\right]^{R_{q-1}} \int_{x_{q-1}}^{x_{q+1}} x_{q}^{i+1}\left[1-F\left(x_{q}\right)\right]^{R_{q}+1} d x_{q} \times \\
f\left(x_{q+1}\right)\left[1-F\left(x_{q+1}\right)\right]^{R_{q+1}} \ldots f\left(x_{m}\right)\left[1-F\left(x_{m}\right)\right]^{R_{m}} d x_{r+1} \ldots d x_{q-1} d x_{q+1} \ldots d x_{m} \\
+\frac{\left(n-R_{r+1}-\cdots-R_{q}-q\right)}{R_{q}+1} \mu_{q: m-1: n}^{\left(R_{r+1}, \ldots R_{q-1},\left(R_{q}+R_{q+1}+1\right), R_{q+2}, \ldots, R_{m}\right)^{(i+2)}} \\
-\frac{\left(n-R_{r+1}-\cdots-R_{q-1}-q+1\right)}{R_{q}+1} \mu_{q-1: m-1: n}^{\left(R_{r+1}, \ldots R_{q-2},\left(R_{q-1}+R_{q}+1\right), R_{q+1}, \ldots, R_{m}\right)^{(i+2)}}
\end{gathered}
$$

Now by substituting for $\mu_{q: m: n}^{\left(R_{r+1}, \ldots, R_{m}\right)^{(i+1)}}$ and $\mu_{q: m: n}^{\left(R_{r+1}, \ldots, R_{m}\right)^{(i+2)}}$ from Eq. (23) and Eq. (24) in Eq. (19), we get

$$
\begin{gathered}
\mu_{q: m: n}^{\left(R_{r+1}, \ldots, R_{m}\right)^{(i)}}=A_{(n, m-1)} \iint \ldots \int_{0<x_{r+1}<\cdots<x_{m}<\infty} x_{q}^{i}\left[F\left(x_{r+1}\right)\right]^{r}\left(\alpha+\theta x_{q}\right) \times \\
f\left(x_{r+1}\right)\left[1-F\left(x_{r+1}\right)\right]^{R_{r+1}} \ldots f\left(x_{q-1}\right)\left[1-F\left(x_{q-1}\right)\right]^{R-1}\left[1-F\left(x_{q}\right)\right]^{R_{q}+1} \times \\
f\left(x_{q+1}\right)\left[1-F\left(x_{q+1}\right)\right]^{R_{q+1}} \ldots f\left(x_{m}\right)\left[1-F\left(x_{m}\right)\right]^{R_{m}} d x_{r+1} \ldots d x_{m}
\end{gathered}
$$

We get

$$
\begin{gathered}
A_{(n, m-1)} \iint \ldots \int_{0<x_{r+1}<\cdots<x_{m}<\infty} x_{q}^{i}\left[F\left(x_{r+1}\right)\right]^{r}\left[f\left(x_{q}\right)-\left(\alpha+\theta x_{q}\right)\left[1-F\left(x_{q}\right)\right]\right] \times \\
{\left[1-F\left(x_{q}\right)\right]^{R_{q}} f\left(x_{r+1}\right)\left[1-F\left(x_{r+1}\right)\right]^{R_{r+1}} \ldots f\left(x_{q-1}\right)\left[1-F\left(x_{q-1}\right)\right]^{R_{q-1}} \times} \\
f\left(x_{q+1}\right)\left[1-F\left(x_{q+1}\right)\right]^{R_{q+1}} \ldots f\left(x_{m}\right)\left[1-F\left(x_{m}\right)\right]^{R_{m}} d x_{r+1} \ldots d x_{m}=0
\end{gathered}
$$

Using Muntz-Szasz theorem, (See, Hwang and Lin [10]), we get

$$
f\left(x_{q}\right)=\left(\alpha+\theta x_{q}\right)\left[1-F\left(x_{q}\right)\right]
$$

Using Theorem 3.1, we get

$$
F(x)=1-e^{-\alpha x-\frac{\theta x^{2}}{2}}
$$

That is the distribution function of linear failure rate distribution.

This completes the proof.

$$
\begin{gathered}
\mu_{q, s: m: n}^{\left(R_{r+1}, \ldots, R_{m}\right)^{(i+2, j)}}=\frac{(i+2)}{\theta\left(R_{q}+1\right)} \mu_{q, s: m: n}^{\left(R_{r+1}, \ldots, R_{m}\right)^{(i, j)}}-\frac{\alpha(i+2)}{\theta(i+1)} \mu_{q, s: m: n}^{\left(R_{r+1}, \ldots, R_{m}\right)^{(i+1, j)}} \\
+\frac{\left(n-R_{r+1}-\cdots-R_{q-1}-q+1\right)}{\left(R_{q}+1\right)}\left[\mu_{q-1, s-1: m-1: n}^{\left(R_{r+1}, \ldots, R_{q-2},\left(R_{q-1}+R_{q}+1\right), R_{q+1}, \ldots, R_{m}\right)}\right)^{(i+2, j)} \\
\left.+\frac{\alpha(i+2)}{\theta(i+1)} \mu_{q-1, s-1: m-1: n}^{\left(R_{r+1}, \ldots, R_{q-2},\left(R_{q-1}+R_{q}+1\right), R_{q+1}, \ldots, R_{m}\right)^{(i+1, j)}}\right] \\
-\frac{\left(n-R_{r+1}-\cdots-R_{q}-q\right)}{\left(R_{q}+1\right)}\left[\mu_{q, s-1: m-1: n}^{\left(R_{r+1}, \ldots, R_{q-1},\left(R_{q}+R_{q+1}+1\right), R_{q+2}, \ldots, R_{m}\right)^{(i+2, j)}}+\frac{\alpha(i+2)}{\theta(i+1)} \mu_{q, s-1: m-1: n}^{\left(R_{r+1}, \ldots, R_{q-1},\left(R_{q}+R_{q+1}+1\right), R_{q+2}, \ldots, R_{m}\right)^{(i+1, j)}}\right]
\end{gathered}
$$


Proof

Necessity:

Theorem 2.2 proved the necessary part of this theorem

Sufficiency:

Assuming that equation (26) holds, then we have:

$$
\begin{aligned}
\mu_{q, s: m: n}^{\left(R_{r+1}, \ldots, R_{m}\right)^{(i, j)}=}= & \frac{\theta\left(R_{q}+1\right)}{(i+2)} \mu_{q, s: m: n}^{\left(R_{r+1}, \ldots, R_{m}\right)^{(i+2, j)}}+\frac{\alpha\left(R_{q}+1\right)}{(i+1)} \mu_{q, s: m: n}^{\left(R_{r+1}, \ldots, R_{m}\right)^{(i+1, j)}} \\
+\left(n-R_{r+1}-\cdots-\right. & \left.R_{q}-q\right)\left[\frac{\theta}{(i+2)} \mu_{q, s-1: m-1: n}^{\left(R_{r+1}, \ldots, R_{q-1},\left(R_{q}+R_{q+1}+1\right), R_{q+2}, \ldots, R_{m}\right)^{(i+2, j)}}\right. \\
& +\frac{\alpha}{(i+1)} \mu_{q, s-1: m-1: n}^{\left.\left(R_{r+1}, \ldots, R_{q-1},\left(R_{q}+R_{q+1}+1\right), R_{q+2}, \ldots, R_{m}\right)^{(i+1, j)}\right]} \\
& -\left(n-R_{r+1}-\cdots-R_{q-1}-q+1\right)\left[\frac{\theta}{(i+2)} \mu_{q-1, s-1: m-1: n}^{\left(R_{r+1}, \ldots, R_{q-2},\left(R_{q-1}+R_{q}+1\right), R_{q+1}, \ldots, R_{m}\right)^{(i+2, j)}}+\right. \\
& \left.\frac{\alpha}{(i+1)} \mu_{q-1, s-1: m-1: n}^{\left(R_{r+1}, \ldots, R_{q-2},\left(R_{q-1}+R_{q}+1\right), R_{q+1}, \ldots, R_{m}\right)^{(i+1, j)}}\right]
\end{aligned}
$$

where

$$
\begin{gathered}
\mu_{q, s: m: n}^{\left(R_{r+1}, \ldots, R_{m}\right)^{(i+1, j)}}=A_{(n, m-1)} \iint \ldots \int_{0<x_{r+1}<\cdots<x_{q-1}<x_{q+1}<\cdots<x_{m}<\infty}\left[F\left(x_{r+1}\right)\right]^{r} \times \\
I_{3}\left(x_{q-1}, x_{q+1}\right) x_{s}^{j} f\left(x_{r+1}\right)\left[1-F\left(x_{r+1}\right)\right]^{R_{r+1}} \ldots f\left(x_{q-1}\right)\left[1-F\left(x_{q-1}\right)\right]^{R_{q-1}} \times \\
f\left(x_{q+1}\right)\left[1-F\left(x_{q+1}\right)\right]^{R_{q+1}} \ldots f\left(x_{m}\right)\left[1-F\left(x_{m}\right)\right]^{R_{m}} d x_{r+1} \ldots d x_{q-1} d x_{q+1} \ldots d x_{m}
\end{gathered}
$$

Substituting by Eq. (22) in Eq. (28), we get

$$
\begin{aligned}
& \mu_{q, s: m: n}^{\left(R_{r+1}, \ldots, R_{m}\right)^{(i+1, j)}}=A_{(n, m-1)} \frac{i+1}{R_{q}+1} \iint \ldots \int_{0<x_{r+1}<\cdots<x_{q-1}<x_{q+1}<\cdots<x_{m}<\infty} x_{s}^{j}\left[F\left(x_{r+1}\right)\right]^{r} \times \\
& f\left(x_{r+1}\right)\left[1-F\left(x_{r+1}\right)\right]^{R_{r+1}} \ldots f\left(x_{q-1}\right)\left[1-F\left(x_{q-1}\right)\right]^{R_{q-1}} \int_{x_{q-1}}^{x_{q+1}} x_{q}^{i}\left[1-F\left(x_{q}\right)\right]^{R_{q}+1} d x_{q} \times \\
& f\left(x_{q+1}\right)\left[1-F\left(x_{q+1}\right)\right]^{R q+1} \ldots f\left(x_{m}\right)\left[1-F\left(x_{m}\right)\right]^{R_{m}} d x_{r+1} \ldots d x_{q-1} d x_{q+1} \ldots d x_{m} \\
& +\frac{A_{(n, m-1)}}{R_{q}+1} \iint \cdots \int_{0<x_{r+1}<\cdots<x_{q-1}<x_{q+1}<\cdots<x_{m}<\infty} x_{q-1}^{i+1} x_{s}^{j}\left[F\left(x_{r+1}\right)\right]^{r} \times \\
& f\left(x_{r+1}\right)\left[1-F\left(x_{r+1}\right)\right]^{R_{r+1}} \ldots f\left(x_{q-1}\right)\left[1-F\left(x_{q-1}\right)\right]^{1+R_{q}+R_{q-1}} f\left(x_{q+1}\right)\left[1-F\left(x_{q+1}\right)\right]^{R_{q+1}} \times \\
& \ldots f\left(x_{m}\right)\left[1-F\left(x_{m}\right)\right]^{R} m d x_{r+1} \ldots d x_{q-1} d x_{q+1} \ldots d x_{m} \\
& -\frac{A_{(n, m-1)}}{R_{q}+1} \iint \cdots \int_{0<x_{r+1}<\cdots<x_{q-1}<x_{q+1}<\cdots<x_{m}<\infty} x_{q+1}^{i+1} x_{s}^{j}\left[F\left(x_{r+1}\right)\right]^{r} \times \\
& f\left(x_{r+1}\right)\left[1-F\left(x_{r+1}\right)\right]^{R_{r+1}} \ldots f\left(x_{q-1}\right)\left[1-F\left(x_{q-1}\right)\right]^{R_{q-1}} f\left(x_{q+1}\right)\left[1-F\left(x_{q+1}\right)\right]^{1+R_{q}+R_{q+1}} \times \\
& \ldots f\left(x_{m}\right)\left[1-F\left(x_{m}\right)\right]^{R m} d x_{r+1} \ldots d x_{q-1} d x_{q+1} \ldots d x_{m} \\
& =A_{(n, m-1)} \frac{i+1}{R_{q}+1} \iint \cdots \int_{0<x_{r+1}<\cdots<x_{q-1}<x_{q+1}<\cdots<x_{m}<\infty} x_{s}^{j}\left[F\left(x_{r+1}\right)\right]^{r} \times
\end{aligned}
$$




$$
\begin{gathered}
f\left(x_{r+1}\right)\left[1-F\left(x_{r+1}\right)\right]^{R_{r+1}} \ldots f\left(x_{q-1}\right)\left[1-F\left(x_{q-1}\right)\right]^{R_{q-1}} \int_{x_{q-1}}^{x_{q+1}} x_{q}^{i}\left[1-F\left(x_{q}\right)\right]^{R_{q}+1} d x_{q} \times \\
f\left(x_{q+1}\right)\left[1-F\left(x_{q+1}\right)\right]^{R_{q+1}} \ldots f\left(x_{m}\right)\left[1-F\left(x_{m}\right)\right]^{R_{m}} d x_{r+1} \ldots d x_{q-1} d x_{q+1} \ldots d x_{m}+ \\
\left.\frac{\left(n-R_{r+1}-\cdots-R_{q-1}-q+1\right)}{R_{q}+1} \mu_{q-1, s-1: m-1: n}^{\left(R_{r+1}, \ldots, R_{q-2},\left(R_{q-1}+R_{q}+1\right), R_{q+1}, \ldots, R_{m}\right)}\right)^{(i+1, j)} \\
-\frac{\left(n-R_{r+1}-\cdots-R_{q}-q\right)}{R_{q}+1} \mu_{q, s-1: m-1: n}^{\left(R_{r+1}, \ldots, R_{q-1},\left(R_{q}+R_{q+1}+1\right), R_{q+2}, \ldots, R_{m}\right)}
\end{gathered}
$$

and

$$
\begin{gathered}
\mu_{q, s: m: n}^{\left(R_{r+1}, \ldots, R_{m}\right)^{(i+2, j)}}=\frac{i+2}{R_{q}+1} A_{(n, m-1)} \iint \ldots \int_{0<x_{r+1}<\cdots<x_{q-1}<x_{q+1}<\cdots<x_{m}<\infty} x_{s}^{j}\left[F\left(x_{r+1}\right)\right]^{r} \times \\
f\left(x_{r+1}\right)\left[1-F\left(x_{r+1}\right)\right]^{R_{r+1}} \ldots \int_{x_{q-1}}^{x_{q+1}} x_{q}^{i+1}\left[1-F\left(x_{q}\right)\right]^{R_{q}+1} d x_{q} f\left(x_{q-1}\right)\left[1-F\left(x_{q-1}\right)\right]^{R_{q-1}} \\
f\left(x_{q+1}\right)\left[1-F\left(x_{q+1}\right)\right]^{R_{q+1}} \ldots f\left(x_{m}\right)\left[1-F\left(x_{m}\right)\right]^{R_{m}} d x_{r+1} \ldots d x_{q-1} d x_{q+1} \ldots d x_{m} \\
\left.+\frac{\left(n-R_{r+1}-\cdots-R_{q-1}-q+1\right)}{R_{q}+1} \mu_{q-1, s-1: m-1: n}^{\left(R_{r+1}, \ldots, R_{q-2},\left(R_{q-1}+R_{q}+1\right), R_{q+1}, \ldots, R_{m}\right)}\right)^{(i+2, j)} \\
-\frac{\left(n-R_{r+1}-\cdots-R_{q}-q\right)}{R_{q}+1} \mu_{q, s-1: m-1: n}^{\left(R_{r+1}, \ldots, R_{q-1},\left(R_{q}+R_{q+1}+1\right), R_{q+2}, \ldots, R_{m}\right)^{(i+2, j)}}
\end{gathered}
$$

Now $\mu_{q, S: m: n}^{\left(R_{r+1, \ldots, R_{m}}\right)^{(i+1, j)}}$

by substituting for (30) in Eq. (27), we get and $\left.\mu_{q, s: m: n}^{\left(R_{r+1}, \ldots, R_{m}\right)}\right)^{(i+2, j)}$ from Eq. (29) and Eq.

$$
\begin{gathered}
\mu_{q, s: m: n}^{\left(R_{r+1}, \ldots, R_{m}\right)^{(i, j)}}=A_{(n, m-1)} \iint \ldots \int_{0<x_{r+1}<\cdots<x_{m}<\infty} x_{q}^{i} x_{s}^{j}\left[F\left(x_{r+1}\right)\right]^{r}\left(\alpha+\theta x_{q}\right) \\
f\left(x_{r+1}\right)\left[1-F\left(x_{r+1}\right)\right]^{R_{r+1}} \ldots f\left(x_{q-1}\right)\left[1-F\left(x_{q-1}\right)\right]^{R_{q-1}}\left[1-F\left(x_{q}\right)\right]^{R_{q}+1} \times \\
f\left(x_{q+1}\right)\left[1-F\left(x_{q+1}\right)\right]^{R_{q+1}} \ldots f\left(x_{m}\right)\left[1-F\left(x_{m}\right)\right]^{R_{m}} d x_{r+1} \ldots d x_{m}
\end{gathered}
$$

Then

$$
\begin{aligned}
& A_{(n, m-1)} \iint \ldots \int_{0<x_{r+1}<\cdots<x_{m}<\infty} x_{q}^{i} x_{s}^{j}\left[F\left(x_{r+1}\right)\right]^{r}\left[f\left(x_{q}\right)-\left(\alpha+\theta x_{q}\right)\left[1-F\left(x_{q}\right)\right]\right] \times \\
& {\left[1-F\left(x_{q}\right)\right]^{R_{q}} f\left(x_{r+1}\right)\left[1-F\left(x_{r+1}\right)\right]^{R_{r+1}} \ldots f\left(x_{q-1}\right)\left[1-F\left(x_{q-1}\right)\right]^{R_{q-1}} \times} \\
& f\left(x_{q+1}\right)\left[1-F\left(x_{q+1}\right)\right]^{R+1} \ldots f\left(x_{m}\right)\left[1-F\left(x_{m}\right)\right]^{R_{m}} d x_{r+1} \ldots d x_{m}=0
\end{aligned}
$$

Using Muntz-Szasz theorem, (See, Hwang and Lin [10]), we get

$$
f\left(x_{q}\right)=\left(\alpha+\theta x_{q}\right)\left[1-F\left(x_{q}\right)\right]
$$

Using Theorem 3.1, we get

$$
\begin{gathered}
F(x)=1-e^{-\alpha x-\frac{\theta x^{2}}{2}} \quad \begin{array}{l}
\text { distribution } \\
n \text { and } i, j \geq 0,
\end{array} \quad \text { iff, for } r+ \\
\mu_{r, s: m: n}^{\left(R_{r+1}, \ldots, R_{m}\right)^{(i, j+2)}}=\frac{(j+2)}{\theta\left(R_{s}+1\right)} \mu_{q, s: m: n}^{\left(R_{r+1}, \ldots, R_{m}\right)^{(i, j)}}-\frac{\alpha(j+2)}{\theta(j+1)} \mu_{q, s: m: n}^{\left(R_{r+1}, \ldots, R_{m}\right)^{(i, j+1)}}
\end{gathered}
$$

That is the distribution function of linear failure rate distribution.

This completes the proof.

Theorem 3.4

Let $X_{r+1: n} \leq X_{r+2: n} \leq \ldots \leq X_{m: n}$ be the order stasistics of a random sample of size $(n-r)$. Then $X$ has linear failure rate distribution iff, for $\quad r+1 \leq q<s \leq m-1, m \leq$ 


$$
\begin{array}{r}
+\frac{\left(n-R_{r+1}-\cdots-R_{S-1}-s+1\right)}{\left(R_{S}+1\right)}\left[\mu_{q, S-1: m-1: n}^{\left(R_{r+1}, \ldots, R_{S-2},\left(R_{S-1}+R_{S}+1\right), R_{S+1}, \ldots, R_{m}\right)^{(i, j+2)}}+\frac{\alpha(j+2)}{\theta(j+1)} \mu_{q, S-1: m-1: n}^{\left(R_{r+1}, \ldots, R_{S-2},\left(R_{S-1}+R_{S}+1\right), R_{S+1}, \ldots, R_{m}\right)^{(i, j+1)}}\right] \\
-\frac{\left(n-R_{r+1}-\cdots-R_{S}-s\right)}{\left(R_{S}+1\right)}\left[\mu_{q, s: m-1: n}^{\left(R_{r+1}, \ldots, R_{S-1},\left(R_{S}+R_{S+1}+1\right), R_{S+2}, \ldots, R_{m}\right)^{(i, j+2)}}+\frac{\alpha(j+2)}{\theta(j+1)} \mu_{q, s: m-1: n}^{\left(R_{r+1}, \ldots, R_{S-1},\left(R_{S}+R_{S+1}+1\right), R_{S+2}, \ldots, R_{m}\right)^{(i, j+1)}}\right]
\end{array}
$$

Proof

Necessity:

Theorem 2.3 proved the necessary part of this theorem.

Sufficiency:

Assuming that equation (32) holds, then we have:

$$
\begin{aligned}
& \mu_{q, s: m: n}^{\left(R_{r+1}, \ldots, R_{m}\right)^{(i, j)}}=\frac{\theta\left(R_{s}+1\right)}{(j+2)} \mu_{q, s: m: n}^{\left(R_{r+1}, \ldots, R_{m}\right)^{(i, j+2)}}+\frac{\alpha\left(R_{s}+1\right)}{(j+1)} \mu_{q, s: m: n}^{\left(R_{r+1}, \ldots, R_{m}\right)^{(i, j+1)}} \\
& +\left(n-R_{r+1}-\cdots-R_{S}-s\right)\left[\frac{\theta}{(j+2)} \mu_{q, S-1: m-1: n}^{\left(R_{r+1}, \ldots, R_{S-1},\left(R_{S}+R_{S+1}+1\right), R_{S+2}, \ldots, R_{m}\right)^{(i, j+2)}}+\frac{\alpha}{(j+1)} \mu_{q, s-1: m-1: n}^{\left(R_{r+1}, \ldots, R_{S-1},\left(R_{S}+R_{S+1}+1\right), R_{S+2}, \ldots, R_{m}\right)^{(i, j+1)}}\right] \\
& -\left(n-R_{r+1}-\cdots-R_{s-1}-s+1\right)\left[\begin{array}{c}
\frac{\theta}{(j+2)} \mu_{q-1, S-1: m-1: n}^{\left(R_{r+1}, \ldots, R_{S-2},\left(R_{S-1}+R_{S}+1\right), R_{S+1}, \ldots, R_{m}\right)^{(i, j+2)}} \\
+\frac{\alpha}{(j+1)} \mu_{q, S-1: m-1: n}^{\left(R_{r+1}, \ldots, R_{S-2},\left(R_{S-1}+R_{S}+1\right), R_{S+1}, \ldots, R_{m}\right)^{(i, j+1)}}
\end{array}\right]
\end{aligned}
$$

From Eq. (4) and Eq. (6), we get

$$
\begin{gathered}
\mu_{q, s: m: n}^{\left(R_{r+1}, \ldots, R_{m}\right)^{(i, j+1)}}=A_{(n, m-1)} \iint \ldots \int_{0<x_{r+1}<\cdots<x_{s-1}<x_{s+1}<\cdots<x_{m}<\infty}\left[F\left(x_{r+1}\right)\right]^{r} x_{q}^{i} \times \\
I_{4}\left(x_{s-1}, x_{s+1}\right) f\left(x_{r+1}\right)\left[1-F\left(x_{r+1}\right)\right]^{R_{r+1}} f\left(x_{s-1}\right)\left[1-F\left(x_{s-1}\right)\right]^{R_{s-1}} \times \\
f\left(x_{s+1}\right)\left[1-F\left(x_{s+1}\right)\right]^{R_{s+1}} \ldots f\left(x_{m}\right)\left[1-F\left(x_{m}\right)\right]^{R_{m}} d x_{r+1} \ldots d x_{s-1} d x_{s+1} \ldots d x_{m}
\end{gathered}
$$

where

$$
I_{4}\left(x_{s-1}, x_{s+1}\right)=\int_{x_{s-1}}^{x_{s+1}} x_{s}^{j+1} f\left(x_{s}\right)\left[1-F\left(x_{s}\right)\right]^{R_{s}} d x_{s}
$$

Integrating by parts, we have obtain

$$
\begin{aligned}
I_{4}\left(x_{s-1}, x_{s+1}\right)= & \frac{-1}{R_{s}+1} x_{s+1}^{j+1}\left[1-F\left(x_{s+1}\right)\right]^{R_{s}+1}+\frac{x_{s-1}^{j+1}}{1+R_{s}}\left[1-F\left(x_{s-1}\right)\right]^{R_{S}+1} \\
& +\frac{i+1}{R_{S}+1} \int_{x_{s-1}}^{x_{s+1}} x_{s}^{j}\left[1-F\left(x_{s}\right)\right]^{R_{S}+1} d x_{s}
\end{aligned}
$$

Now by substituting in Eq. (34), we get

$$
\begin{gathered}
\mu_{q, s: m: n}^{\left(R_{r+1}, \ldots, R_{m}\right)^{(i, j+1)}}=A_{(n, m-1)} \frac{j+1}{R_{s}+1} \iint \ldots \int_{0<x_{r+1}<\cdots<x_{s-1}<x_{s+1}<\cdots<x_{m}<\infty} x_{q}^{i}\left[F\left(x_{r+1}\right)\right]^{r} \times \\
f\left(x_{r+1}\right)\left[1-F\left(x_{r+1}\right)\right]^{R_{r+1}} \ldots f\left(x_{s-1}\right)\left[1-F\left(x_{s-1}\right)\right]^{R_{s-1}} \int_{x_{s-1}}^{x_{s+1}} x_{s}^{j}\left[1-F\left(x_{s}\right)\right]^{1+R_{s}} d x_{s} \times \\
f\left(x_{s+1}\right)\left[1-F\left(x_{s+1}\right)\right]^{R_{s+1}} \ldots f\left(x_{m}\right)\left[1-F\left(x_{m}\right)\right]^{R_{m}} d x_{r+1} \ldots d x_{s-1} d x_{s+1} \ldots d x_{m} \\
+\frac{A_{(n, m-1)}}{R_{s}+1} \iint \ldots \int_{0<x_{r+1}<\cdots<x_{s-1}<x_{s+1}<\cdots<x_{m}<\infty} x_{s-1}^{j+1} x_{q q}^{i}\left[F\left(x_{r+1}\right)\right]^{r} \times \\
f\left(x_{r+1}\right)\left[1-F\left(x_{r+1}\right)\right]^{R+1} \ldots f\left(x_{s-1}\right)\left[1-F\left(x_{s-1}\right)\right]^{1+R_{s}+R_{s-1}} f\left(x_{s+1}\right)\left[1-F\left(x_{s+1}\right)\right]^{R_{s+1} \ldots \times} \times \\
f\left(x_{m}\right)\left[1-F\left(x_{m}\right)\right]^{R_{m}} d x_{r+1} \ldots d x_{s-1} d x_{s+1} \ldots d x_{m}
\end{gathered}
$$




$$
\begin{aligned}
& -\frac{A_{(n, m-1)}}{R_{S}+1} \iint \cdots \int_{0<x_{r+1}<\cdots<x_{s-1}<x_{s+1}<\cdots<x_{m}<\infty} x_{s+1}^{j+1} x_{q}^{i}\left[F\left(x_{r+1}\right)\right]^{r} \times \\
& f\left(x_{r+1}\right)\left[1-F\left(x_{r+1}\right)\right]^{R_{r+1}} \ldots f\left(x_{s-1}\right)\left[1-F\left(x_{s-1}\right)\right]^{R_{S-1}} f\left(x_{s+1}\right)\left[1-F\left(x_{s+1}\right)\right]^{1+R_{S}+R_{s+1}} \ldots \times \\
& f\left(x_{m}\right)\left[1-F\left(x_{m}\right)\right]^{R_{m}} d x_{r+1} \ldots d x_{s-1} d x_{s+1} \ldots d x_{m} \\
& =A_{(n, m-1)} \frac{j+1}{R_{s}+1} \iint \cdots \int_{0<x_{r+1}<\cdots<x_{s-1}<x_{s+1}<\cdots<x_{m}<\infty}\left[F\left(x_{r+1}\right)\right]^{r} \times \\
& f\left(x_{r+1}\right)\left[1-F\left(x_{r+1}\right)\right]^{R_{r+1}} \ldots f\left(x_{s-1}\right)\left[1-F\left(x_{s-1}\right)\right]^{R_{s-1}} \int_{x_{s-1}}^{x_{s+1}} x_{s}^{j} x_{q}^{i}\left[1-F\left(x_{s}\right)\right]^{R_{s}+1} d x_{s} \\
& f\left(x_{s+1}\right)\left[1-F\left(x_{s+1}\right)\right]^{R_{S+1}} \ldots f\left(x_{m}\right)\left[1-F\left(x_{m}\right)\right]^{R_{m}} d x_{r+1} \ldots d x_{s-1} d x_{s+1} \ldots d x_{m}+ \\
& \frac{\left(n-R_{r+1}-\cdots-R_{s-1}-s+1\right)}{R_{S}+1} \mu_{q, S-1: m-1: n}^{\left(R_{r+1}, \ldots, R_{S-2},\left(R_{S-1}+R_{S}+1\right), R_{S+1}, \ldots, R_{m}\right)^{(i, j+1)}} \\
& -\frac{\left(n-R_{r+1}-\cdots-R_{S}-s\right)}{R_{S}+1} \mu_{q, S-1: m-1: n}^{\left(R_{r+1}, \ldots, R_{S-1},\left(R_{S}+R_{S+1}+1\right), R_{S+2}, \ldots, R_{m}\right)^{(i, j+1)}}
\end{aligned}
$$

and

$$
\begin{aligned}
& \mu_{q, s: m: n}^{\left(R_{r+1, \ldots, R_{m}}\right)^{(i, j+2)}}=A_{(n, m-1)} \frac{j+2}{R_{s}+1} \iint \ldots \int_{0<x_{r+1}<\cdots<x_{s-1}<x_{s+1}<\cdots<x_{m}<\infty} x_{q}^{i}\left[F\left(x_{r+1}\right)\right]^{r} \times \\
& f\left(x_{r+1}\right)\left[1-F\left(x_{r+1}\right)\right]^{R_{r+1}} \ldots f\left(x_{s-1}\right)\left[1-F\left(x_{s-1}\right)\right]^{R_{s-1}} \int_{x_{s-1}}^{x_{s+1}} x_{s}^{j+1}\left[1-F\left(x_{s}\right)\right]^{R_{s}+1} d x_{s} \\
& f\left(x_{s+1}\right)\left[1-F\left(x_{s+1}\right)\right]^{R_{s+1}} \ldots f\left(x_{m}\right)\left[1-F\left(x_{m}\right)\right]^{R_{m}} d x_{r+1} \ldots d x_{s-1} d x_{s+1} \ldots d x_{m} \\
& +\frac{\left(n-R_{r+1}-\cdots-R_{s-1}-s+1\right)}{R_{s}+1} \mu_{q, s-1: m-1: n}^{\left(R_{r+1}, \ldots, R_{s-2},\left(R_{S-1}+R_{S}+1\right), R_{S+1}, \ldots, R_{m}\right)^{(i, j+2)}} \\
& -\frac{\left(n-R_{r+1}-\cdots-R_{S}-s\right)}{R_{S}+1} \mu_{q, S-1: m-1: n}^{\left(R_{r+1}, \ldots, R_{S-1},\left(R_{S}+R_{S+1}+1\right), R_{S+2}, \ldots, R_{m}\right)^{(i, j+2)}}
\end{aligned}
$$

Now by substituting for $\mu_{q, s: m: n}^{\left(R_{r+1}, \ldots, R_{m}\right)^{(i, j+1)}}$ and $\mu_{q, s: m: n}^{\left(R_{r+1}, \ldots, R_{m}\right)^{(i, j+2)}}$ from Eq. (37) and Eq. (38) in Eq. (33), we get

$$
\begin{gathered}
\mu_{q, s: m: n}^{\left(R_{r+1, \ldots, R_{m}}\right)^{(i, j)}}=A_{(n, m-1)} \iint \ldots \int_{0<x_{r+1}<\cdots<x_{m}<\infty} x_{q}^{i} x_{s}^{j}\left[F\left(x_{r+1}\right)\right]^{r}\left(\alpha+\theta x_{s}\right) \\
f\left(x_{r+1}\right)\left[1-F\left(x_{r+1}\right)\right]^{R_{r+1}} \ldots f\left(x_{s-1}\right)\left[1-F\left(x_{s-1}\right)\right]^{R_{s-1}}\left[1-F\left(x_{s}\right)\right]^{R_{s}+1} \times \\
f\left(x_{s+1}\right)\left[1-F\left(x_{s+1}\right)\right]^{R_{s+1}} \ldots f\left(x_{m}\right)\left[1-F\left(x_{m}\right)\right]^{R_{m}} d x_{r+1} \ldots d x_{m}
\end{gathered}
$$

We get

$$
\begin{gathered}
A_{(n, m-1)} \iint \ldots \int_{0<x_{r+1}<\cdots<x_{m}<\infty} x_{q}^{i} x_{s}^{j}\left[F\left(x_{r+1}\right)\right]^{r}\left[f\left(x_{q}\right)-\left(\alpha+\theta x_{q}\right)\left[1-F\left(x_{q}\right)\right]\right] \times \\
{\left[1-F\left(x_{s}\right)\right]^{R_{s}} f\left(x_{r+1}\right)\left[1-F\left(x_{r+1}\right)\right]^{R_{r+1}} \ldots f\left(x_{s-1}\right)\left[1-F\left(x_{s-1}\right)\right]^{R_{s-1}} f \times} \\
\left(x_{s+1}\right)\left[1-F\left(x_{s+1}\right)\right]^{R_{s+1}} \ldots f\left(x_{m}\right)\left[1-F\left(x_{m}\right)\right]^{R_{m}} d x_{r+1} \ldots d x_{m}=0
\end{gathered}
$$

Using Muntz-Szasz theorem, (See, Hwang and Lin [10]), we get

$$
F(x)=1-e^{-\alpha x-\frac{\theta x^{2}}{2}}
$$

$$
f\left(x_{s}\right)=\left(\alpha+\theta x_{s}\right)\left[1-F\left(x_{s}\right)\right]
$$

Using Theorem 3.1 above, we get

That is the distribution function of linear failure rate distribution.

This completes the proof. 


\section{Conclusion}

We derived recurrence relations among single and product moments of general progressively Type-II right censored order statistics (Theorem 2.1, 2.2 and 2.3). Characterization for the random variable $X$ following the linear failure rate distribution is obtained using the previous recurrence relations (Theorems 3.2, 3.3 and 3.4) and distribution function (Theorem 3.1). For future work, estimation for the scale and location parameters could be obtained by applying the best linear unbiased estimation to the previous results.

\section{Acknowledgements}

The authors wish to thank the referees for suggesting some changes which led to an improvement in the presentation of this paper.

\section{References}

[1] Abd El-Aty, Y. and Marwa M. Mohie El-Din. Recurrence relations for single and double moments of GOS from the inverted linear exponential distribution and any continuous function. $J$. of probability and statist. science, 2009; 7:127137.

[2] Abd El-Hamid, A. H. and AL-Hussaini, E. K. Inference and optimal design based on step-partially accelerated life tests for the generalized Pareto distribution under progressive Type-I censoring. Communications in Statistics- Simulation and Computation, 2015; 44:1750-1769.

[3] Aggarwala, R. and Balakrishnan, N. Recurrence relations for single and product moments of progressive Type-II right censored order statistics from exponential and truncated exponential distributions. Ann. Inst. Statist. Math., 1996; 4:757-771.

[4] Athar, H. Akhterand, Z. and Saran, J. Moments of progressive
Type-II right censored order statistics from Lindley distribution. Statist. Research Letters, 2014; 3:1-6.

[5] Bain, L. J. Analysis for the Linear Failure-Rate Life-Testing Distribution. Technometrics, 1974; 16:551-559.

[6] Balakrishnan, N., Cramer, E., Kamps, U., Schenk, N. Progressive Type II censored order statistics from exponential distributions. Statistics, 2001; 35:537-556.

[7] Balakrishnan, N. and Sandhu R. A. Best linear unbiased and maximum likelihood estimation for exponential distributions based on general progressive Type-II censored samples. Sankhya, 58, Series B., 1996; 1:1-9.

[8] Davis, H. T., Feldstein, M. L. The generalized Pareto law as a model for progressively censored survival data. Biometrika, 1979; 66:299-306.

[9] Jagdish Saran and Narinder Pushkarna. Derived moments of progressive Type-II right censored order statistics from a general class of doubly truncated continuous distributions. $J$. of Statistical Theory and Applications, 2014; 13:162-174.

[10] Hwang, J. S. and Lin, G. D. Extensions of Muntz-Szasz theorem and applications. Analysis, 1984; 4:143-160.

[11] Mohie El-Din, M. M., Amein, M. M. and Hafez, E. H. Statistical inference and characterizations from independent and identical Exponential-Bernoulli mixture distribution. Advanced Research in Statistics and Probability, Issue, 2011; 3:15-31.

[12] Mohie El-Din, M. M. and Kotb, M. S. Recurrence relations for single and product moments of generalized order statistics for modified Burr XII-geometric distribution and characterization. J. of Advanced Research Statistics and Probability, 2011; 1:36-46.

[13] Mokhlis, N. A., Abd El-Aty, Y and Marwa M. Mohie El-Din. Recurrence relations for moments of GOS from MarshallOlkin-Extended Burr XII distribution. international J. of math. and computation, 2010; 8:127-136. 